\title{
Epigenetic Regulation of Werner Syndrome Gene in Age-Related Cataract
}

\author{
Xi Zhu, Guowei Zhang, Lihua Kang, and Huaijin Guan \\ Eye Institute, Affiliated Hospital of Nantong University, Nantong, Jiangsu 226001, China \\ Correspondence should be addressed to Huaijin Guan; gtnantongeye@gmail.com
}

Received 31 December 2014; Revised 11 March 2015; Accepted 13 March 2015

Academic Editor: Jun Zhang

Copyright ( $2015 \mathrm{Xi}$ Zhu et al. This is an open access article distributed under the Creative Commons Attribution License, which permits unrestricted use, distribution, and reproduction in any medium, provided the original work is properly cited.

\begin{abstract}
Purpose. To examine the promoter methylation and histone modification of WRN (Werner syndrome gene), a DNA repair gene, and their relationship with the gene expression in age-related cataract (ARC) lens. Methods. We collected the lenses after cataract surgery from 117ARC patients and 39 age-matched non-ARC. WRN expression, DNA methylation and histone modification around the CpG island were assessed. The methylation status of Human-lens-epithelium cell (HLEB-3) was chemically altered to observe the relationship between methylation and expression of WRN. Results. The WRN expression was significantly decreased in the ARC anterior lens capsules comparing with the control. The CpG island of WRN promoter in the ARC anterior lens capsules displayed hypermethylation comparing with the controls. The WRN promoter was almost fully methylated in the cortex of ARC and control lens. Acetylated $\mathrm{H} 3$ was lower while methylated H3-K9 was higher in ARC anterior lens capsules than that of the controls. The expression of WRN in HLEB-3 increased after demethylation of the cells. Conclusions. A hypermethylation in WRN promoter and altered histone modification in anterior lens capsules might contribute to the ARC mechanism. The data suggest an association of altered DNA repair capability in lens with ARC pathogenesis.
\end{abstract}

\section{Introduction}

Age-related cataract (ARC) is one of the dominant causes of visual impairment in the elderly [1]. The disease can be classified as cortical, nuclear, and posterior subcapsular according to the location of the opacity within the lens [2]. ARC is a complex disease with multiple genetic and environmental risk components, including UV light, sun exposure, vitamin C deficiency, and hypertension [3], but its etiology is not fully understood $[4,5]$.

Oxidative stress has long been recognized as an important mediator of pathophysiology in lens epithelial cells (LECs) and also plays a vital role in the pathogenesis of cataract [6-8]. Recent studies have reported the association between reactive oxygen species- (ROS-) induced DNA damage of LECs and the development of cataract [8-10]. In our previous studies, we have also found that oxidative DNA damage marker, 8-oxoG, was significantly increased in ARC group compared with control group $[11,12]$. Oxidative DNA lesions are repaired by nucleotide excision repair, double-strand break (DSB) repair, and base excision repair $[13,14]$. The
WRN gene plays an important role in aging [15] and is known to function in repair of damaged DNA, particularly in repairing double-strand breaks [16]. The WRN protein belongs to RecQ family and has 1432 amino acids possessing both $3^{\prime} \rightarrow 5^{\prime}$ DNA helicase and $3^{\prime} \rightarrow 5^{\prime}$ DNA exonuclease activities. These biochemical functions are known to have roles in DNA replication, repair of DNA damage, gene transcription, and telomere maintenance [17]. WRN disruption causes Werner's syndrome (WS), an autosomal recessive segmental progeroid syndrome that results in accelerated aging and affects multiple organs and tissues [17]. Most WS patients develop bilateral ocular cataract when they are 20 years old and beyond [18, 19]. Previously, we reported that polymorphisms and copy number variations of WRN are associated with ARC [20,21].

Epigenetics pertains to heritable alterations in gene expression that do not involve modification of the underlying genomic DNA sequence [22]. DNA methylation and histone modifications (including methylation, acetylation, sumoylation, and phosphorylation) are the major epigenetic mechanisms for gene expression [23]. Hypermethylation of 
TABLE 1: Demographic information of study participants.

\begin{tabular}{lcc}
\hline & Control & ARC \\
\hline$N$ & 39 & 117 \\
Age $($ mean \pm SD) & $69.66 \pm 4.51$ & $70.38 \pm 7.72$ \\
Female $N(\%)$ & $21(54.8)$ & $70(60.1)$ \\
Male $N(\%)$ & $18(45.2)$ & $47(39.9)$ \\
Cortical $N(\%)$ & 0 & $39(33.3)$ \\
Nuclear $N(\%)$ & 0 & $39(33.3)$ \\
PSC $N(\%)$ & 0 & $39(33.3)$ \\
\hline
\end{tabular}

promoter $\mathrm{CpG}$ islands and histone $\mathrm{H} 3$ methylated at lysine 9 have been linked to heterochromatin and gene silencing, whereas histone $\mathrm{H} 3$ acetylated is enriched in euchromatic domains and correlates with active gene expressions [24-26]. DNA methylation is a critical regulator of gene expression in the eye and is necessary for the proper development and postmitotic survival of retinal neurons [27]. Aberrant methylation patterns have been associated with age-related macular degeneration, cataract, pterygium, and retinoblastoma [28-32]. Changes in histone modifications have also been observed in experimental models of diabetic retinopathy and glaucoma [22]. A decreased expression of WRN is related to aberrant DNA hypermethylation in various tumors; epigenetic inactivation of this gene may be a biomarker for selection of drugs for the treatment of cancer [33-37].

In this study, we investigated WRN expression in anterior lens capsules and lens cortex of ARC and age-matched controls and analyzed the correlation between epigenetic modification and expression profiles of WRN gene to explore the possible effect of epigenetics on the development of ARC.

\section{Materials and Methods}

2.1. Study Participants. The research followed the tenets of the Declaration of Helsinki. All participants signed the informed consent forms. The study was approved by the Ethics Committee of Affiliated Hospital of Nantong University.

We enrolled 117 ARC patients that consisted of three subgroups: age-related cortical cataract (ARC-C), age-related nuclear cataract (ARC-N), and age-related posterior subcapsular cataract (ARC-P). The criteria for ARC group included (1) opaque ocular lenses, (2) $\geq 50$ years of age, and (3) $C \geq 4$, $N \geq 4$, and $P \geq 4$ according to the lens opacity classification system III (LOCSIII) [38] and excluded (1) complicated cataract due to high myopia, uveitis, ocular trauma, or other known causes and (2) hypertension, diabetes, or other systemic diseases. We enrolled 39 patients with vitreoretinal diseases who received transparent lens extraction as control group. The criteria for the control included (1) transparent ocular lenses and (2) $\geq 50$ years of age and excluded (1) other major eye diseases such as glaucoma, myopia, diabetic retinopathy, and uveitis and (2) hypertension, diabetes, or other systemic diseases. The demographic information for all participants was listed in Table 1.

2.2. Anterior Lens Capsules and Lens Cortex Preparation. Anterior lens capsules that included lens epithelium (LE) and peripheral cortex were obtained through small incision extra capsular cataract surgery. The tissue was dissected and rapidly frozen in liquid nitrogen and then stored at $-80^{\circ} \mathrm{C}$ for protein, RNA, and genomic DNA extraction.

2.3. RNA Isolation and cDNA Preparation. Total RNA from anterior lens capsules and lens cortex was isolated from the frozen tissues using TRIzol reagent (Invitrogen, Carlsbad, CA), and cDNAs were synthesized using PrimeScript RT reagent Kit (TaKaRa, Dalian, China).

2.4. Quantification of WRN mRNA. TaqMan gene expression assay probe (Applied Biosystems, Foster City, CA) was used for WRN mRNA quantification (Applied Biosystems assay ID: Hs01087915_ml). The results were normalized against the expression of housekeeping gene GAPDH from the same sample. RT-PCR was performed using ABI 7500 real time PCR system (Applied Biosystems, Foster City, CA). The fold change of WRN expression was determined using the comparative CT $\left(2^{-\Delta \Delta \mathrm{CT}}\right)$ method.

2.5. Western Blot Assay. The protein of anterior lens capsules and lens cortex was extracted in lysis buffer ( $1 \mathrm{M}$ Tris$\mathrm{HCl}$ at $\mathrm{pH} 7.5,1 \%$ Triton $\mathrm{X}-100,1 \%$ Nonidet $\mathrm{p}-40,10 \%$ SDS, $0.5 \%$ sodium deoxycholate, $0.5 \mathrm{M}$ EDTA, $10 \mathrm{mg} / \mathrm{mL}$ leupeptin, $10 \mathrm{mg} / \mathrm{mL}$ aprotinin, and $1 \mathrm{mM}$ phenylmethylsulfonyl fluoride). Proteins were size-fractionated by sodium dodecyl sulfate-polyacrylamide gel electrophoresis and transferred onto polyvinylidene difluoride filter membranes (Millipore, Bedford, MA). Nonspecific protein binding to the membrane was blocked with blocking buffer (5\% nonfat milk, $200 \mathrm{mM} \mathrm{NaCl}, 50 \mathrm{mM}$ Tris, and 0.05\% Tween 20). The blocked membrane was then incubated with mouse antihuman-WRN (1:800; Abcam, Cambridge, UK) and antiGAPDH (1:1000; Santa Cruz, CA, USA) at $4^{\circ} \mathrm{C}$ for $18 \mathrm{~h}$. The membrane was washed three times with TBST (20 mM Tris, $500 \mathrm{mM} \mathrm{NaCl}$, and $0.1 \%$ Tween 20) for $5 \mathrm{~min}$ each time, followed by incubating with an alkaline phosphatase-conjugated goat anti-mouse IgG antibody (1:2000; Santa Cruz, CA, USA) for $2 \mathrm{~h}$. Detection was performed using an ECL chemiluminescence kit (Pierce, Rockford, IL) and the signal was exposed to an X-ray film that was scanned using Image Quant software (Molecular Dynamics, Sunnyvale, CA, USA).

2.6. DNA Methylation Detection. DNA sequence of WRN from the NCBI genome database was used for the bioinformatic analysis. Transcription start site (TSS) of the gene was predicted by the online database (http://dbtss.hgc.jp/). CpG islands of WRN were predicted by using online software (http://www.urogene.org/methprimer/).

Genomic DNA from the frozen tissues and HLEB-3 was isolated by phenol/chloroform and ethanol extraction. Two micrograms of genomic DNA was treated with sodium bisulfite using the EpiTect Bisulfite Kit (Qiagen, Inc., Frederick, MD). EpiTect Control DNA (Qiagen, Inc.) was used as the positive controls in all experiments.

The bisulfite-sequencing PCR (BSP) primer was designed by web-based Meth Primer software (http://www.urogene .org/methprimer/) to cover a CpG island near WRN. The 
primers used for region 1 were $5^{\prime}$-TTATTTTGAAAGAAGTTTTTTTTGG-3' $3^{\prime}$ (forward) and $5^{\prime}$-AAACAAACTATTATCCTCCCAACAC- $3^{\prime}$ (reverse). The primers used for region 2 were $5^{\prime}$-TTTTTTGTGTTGGGAGGATAATAGT$3^{\prime}$ (forward) and $5^{\prime}$-AACAAAAAACAAAACTCCAAAAAAA- $3^{\prime}$ (reverse). The primers used for region 3 were $5^{\prime}$ AGGTCTCCAGCCGGCGGGCACTCA-3' (forward) and $5^{\prime}$-TGAGGGGAAGAGGGGGTC- ${ }^{\prime}$ (reverse). The primers used for region 4 were $5^{\prime}$-TTTAGTGTATTTTTTGATTGAAGTT- $3^{\prime}$ (forward) and $5^{\prime}$-CTAAACAACTAAAATCCTACATCCC- $3^{\prime}$ (reverse). The PCR products were gelextracted and cloned into the pMD-20-T vector (Takara, Japan). Plasmid-transformed bacteria DH5a were grown for $14 \mathrm{~h}$ and the plasmid DNA was isolated. At least 10 clones were chosen for sequence analysis. The degree of methylation was presented as $\mathrm{mC} / \mathrm{CpG}$.

2.7. Chromatin Immunoprecipitation (ChIP) Assay. Chromatin immunoprecipitation (ChIP) assay was performed using Tissue Acetyl-Histone H3 ChIP kit and Tri-MethylHistone H3K9 ChIP kit (Epigentek, Farmingdale, NY, USA) according to the manufacturer's instructions. Briefly, the anterior lens capsules and cortex were cross-linked with $1 \%$ formaldehyde for $8 \mathrm{~min}$ and then homogenized. The homogenate was sonicated for 4 pulses of $15 \mathrm{sec}$ each at level 2 using the microtip probe of a Branson Digital Sonifier (Model 450, Branson Ultrasonics Corporation, Connecticut, USA), followed by a $40 \mathrm{sec}$ interval on ice between each pulse, to generate fragments of genomic DNA ranging from 200 to $800 \mathrm{bp}$ in length. For the ChIP assays, equal amounts of treated chromatin were added to microwells containing immobilized antibody for the targeted protein or a negative control normal mouse IgG antibody. In addition, a small portion of treated chromatin, which was equal to $5 \%$ of the extracted genomic DNA, was used as the Input DNA to calculate the enrichment of the leptin promoter DNA after immunoprecipitation of the targeted proteins. After incubation for $90 \mathrm{~min}$ at $65^{\circ} \mathrm{C}$ to reverse the crosslinks and elute the DNA, Fast-Spin columns were used for DNA purification. The primers for WRN promoter were $5^{\prime}$-CCGCCGCCTGACTTCGGACACC-3' (forward) and $5^{\prime}$ TCGCACTCCCGCTGCACCCCAC-3' (reverse).

2.8. Cell Culture and Demethylation Treatment. To test the relationship between the methylation and the expression of the WRN, an in vitro study of demethylation was performed. Human lens epithelium cell line (HLEB-3) was obtained from American Type Culture Collection (ATCC; Rockville, MD) and cultured in Eagle's minimum essential medium (Invitrogen-GIBCO, Carlsbad, CA) with $10 \%$ fetal bovine serum at $37^{\circ} \mathrm{C}$ in a humidified $5 \% \mathrm{CO}_{2}$ atmosphere. After reaching $80-90 \%$ confluency, the cells were demethylated by incubation in medium containing $3 \mathrm{mM}$ of 5-aza-2-deoxycytidine (5-aza-dC) (Sigma, CA, USA) for $72 \mathrm{~h}$. Whole cell protein extracts of HLEB-3 were isolated for Western blotting.

2.9. Statistical Analysis. One-way ANOVA analysis was used to determine the difference in averages between the four groups. $P$ value $<0.05$ was considered statistically significant. Statistical analyses were performed with SPSS software (SPSS 17.0; SPSS, Inc., Chicago, IL).

\section{Results}

3.1. Expression of WRN in Anterior Lens Capsules and Lens Cortex. RT-PCR analysis was performed to investigate WRN mRNA content in anterior lens capsules and context of the ARC and the controls. Lower WRN mRNA (Figure 1(a)) expression in anterior lens capsules was detected in all three subtypes of ARC cases compared with the controls $(P<$ 0.01 ).

To confirm the change of WRN in protein level between ARC and controls, Western blot analysis was performed. As shown in Figure 1(b), the expression pattern of WRN protein in anterior lens capsules was lower in all three subtypes of ARC cases compared with the controls $(P<0.01)$. However, WRN was undetected in lens cortex in both the ARC cases and the controls (data not shown).

3.2. Methylation Status of WRN. To analyze the relationship between methylation status and expression of WRN, we detected the methylation rate of WRN promoter in the DNA extracted from the anterior lens capsules and cortex of ARC and control group using BSP. Bioinformatic analysis indicated four $\mathrm{CpG}$ islands in the promoter of WRN (Figure 2(a)) (R1: -1287 to -1133 , R2: -875 to -620 , R3: -209 to 164 , and R4: 173 to 324 , relative to the TSS). Figures 2(b) and 2(c) showed a representative result of bisulfite genomic sequencing of the R3 fragment. Each row stands for a single plasmid clone, and each circle represents a $\mathrm{CpG}$ site. The unmethylated and methylated $\mathrm{CpGs}$ were represented by unfilled and filled cycles, respectively. As shown in Figure 2(b), the methylation rate of all three subtypes of ARC was higher than that of the control group at region 3 of WRN promoter. As shown in Figure 2(c), the methylation rate of lens cortex at region 3 was almost $100 \%$ in both ARC group and control group. The methylation rates at regions 1,2 , and 4 in WRN promoter were almost $100 \%$ in both ARC group and control group (data not shown).

3.3. Histone Modifications around the CPG Islands of the WRN Gene in ARC. In general, hypermethylation of H3-K9 exhibits the silencing of gene expression, whereas acetylation of $\mathrm{H} 3$ is associated with activation of gene expression. We performed ChIP to analyze the correlation between the histone modification and the expression profiles of WRN gene. ChIP analysis depicted that acetylated $\mathrm{H} 3$ levels were lower in all three subtypes of ARC than the control in anterior lens capsules while methylated $\mathrm{H} 3-\mathrm{K} 9$ was increased in all three subtypes of ARC (Figure 3). The histone modification of cortex was undetectable (data not shown).

3.4. Methylation Status and Protein Expression of WRN in HLEB-3 after Treatment with 5-Aza-dC. To test the relationship between the methylation and the expression of the WRN, an in vitro study of demethylation was performed. 5-Aza-dC 


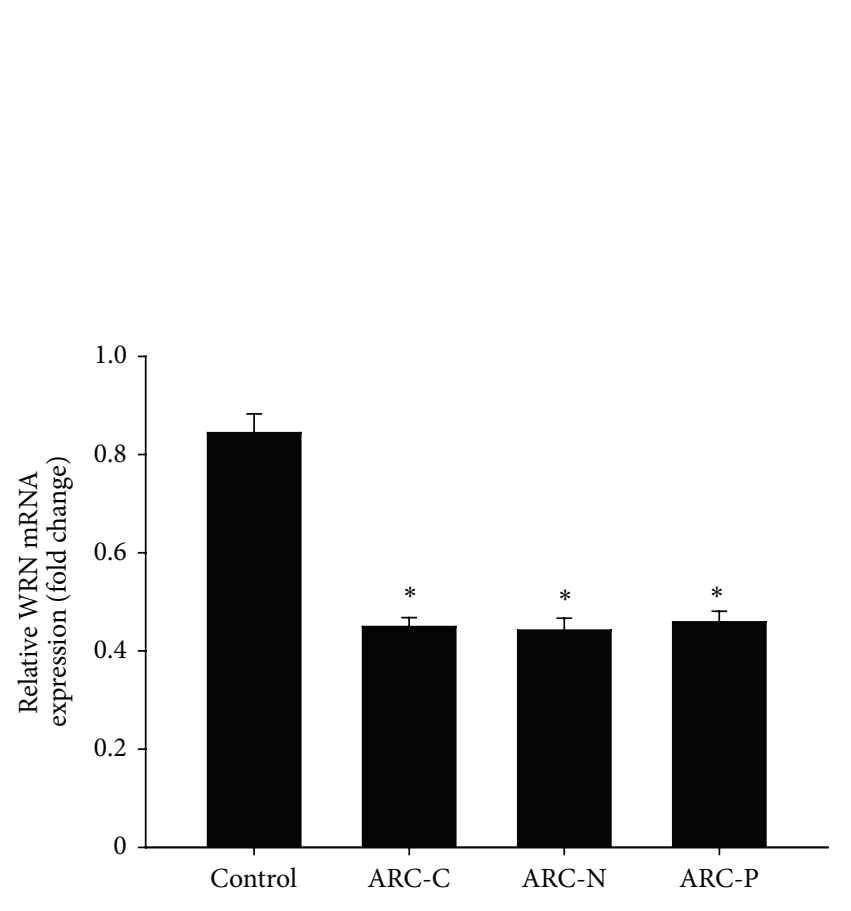

(a)

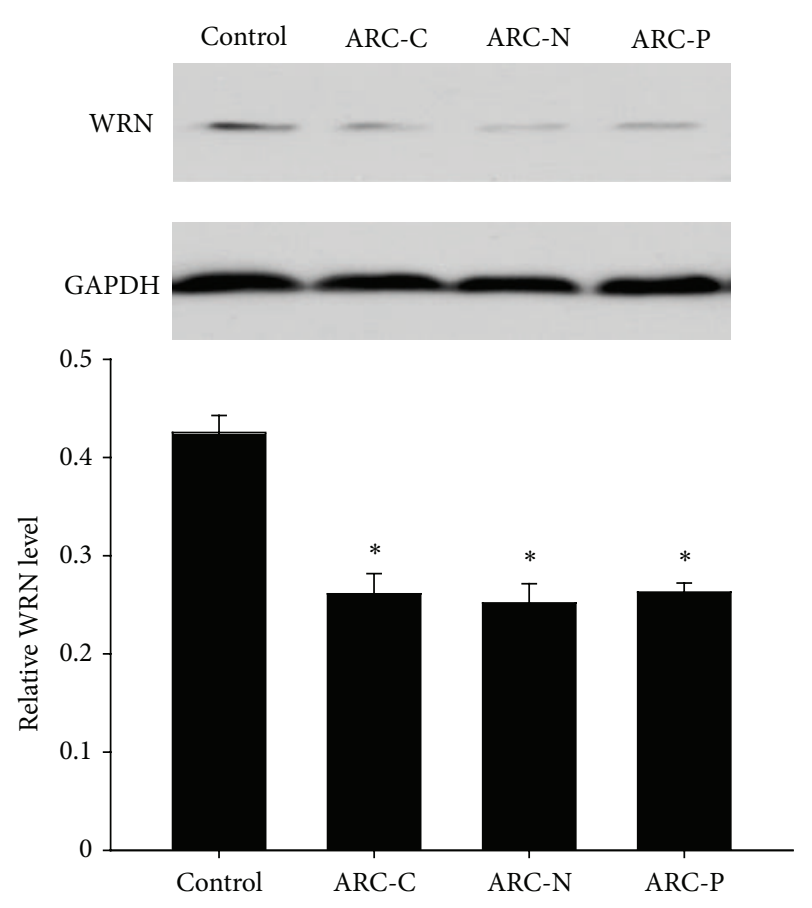

(b)

FIGURE 1: Relative expression of mRNA and protein levels of WRN in anterior lens capsules of control and ARC. (a) RT-PCR analysis of the expression of WRN in control $(n=20)$ and ARC-C $(n=20)$, ARC-N $(n=20)$, and ARC-P $(n=20)$. Values represent mean \pm SD. ${ }^{*} P<0.01$. (b) WRN protein levels in control $(n=9)$ and ARC-C $(n=9)$, ARC-N $(n=9)$, and ARC-P $(n=9)$ anterior lens capsules were detected using Western blotting. Relative WRN protein level to GAPDH is presented as mean \pm SD. ${ }^{*} P<0.01$.

is a DNA methyltransferase inhibitor which is used to inhibit DNA methylation. As shown in Figure 4(a), after treatment with 5-aza-dC, the methylation rate of WRN promoter in HLEB-3 was decreased in comparison with the untreated control. In contrast, the protein expression of WRN in HLEB3 after treatment with 5 -aza-dC was increased in comparison with the untreated control (Figure 4(b)).

\section{Discussion}

Although the pathophysiology of ARC is far from being clearly understood, it is well accepted that oxidative stress plays an important role in the disease pathogenesis. When reactive oxygen species (ROS) production exceeds the capacity of its removal by various mechanisms, they may cause oxidative damage to DNA [6-10]. In a normal physiologic condition, most oxidative DNA lesions are rapidly repaired by base excision repair (BER), nucleotide excision repair (NER), and double-strand break repair (DSBR) pathways $[13,14]$. WRN is a protein functioning in the DSBR pathway and is also required for cellular DNA replication and mismatch repair [33]. Both mRNA and protein expression of WRN are downregulated in anterior lens capsules in ARC, implying a reduced DNA repair capability in the ARC lens from all included subtypes. The results have provided an additional evidence of DNA repair mechanism in ARC development by using patients' lens tissue.

We demonstrated that WRN undergoes epigenetic alterations in ARC lens tissue from all included subtypes and this alteration is associated with the mRNA and protein expression of the gene. The treatment with a demethylating agent restored the WRN expression in HLEB-3. The results linked the epigenetic changes with the target gene expression and are consistent with the current knowledge on the effect of epigenetic modification on human genome.

It is of interest that the ARC-associated epigenetic changes of WRN gene only occur in anterior lens capsules but not in lens cortex in which both ARC cases and control had an undetectable or very weak expression of WRN and a very high degree of WRN methylation. Lens cortex is made up of lens fibers which are differentiated from LECs. The results suggest that the strategies to intervene epigenetic alteration in ARC should aim at anterior lens capsules.

We analyzed methylation status at four regions of WRN promoter in both ARC and control groups; only region 3 showed significant changes between the cases and the controls. This sequence-specific change is reasonable because this region contains the most abundant $\mathrm{CpG}$ islands among the four selected regions and spans the translation starting site.

\section{Conclusions}

Overall, our study found that aberrant epigenetic methylation in WRN DNA and the associated histone linked to low expression of WRN and lens opacity. This is the first report to show a relationship between the epigenetic modification of WRN gene and ARC by directly studying the lens tissue from human subjects. This study provided a deeper insight 


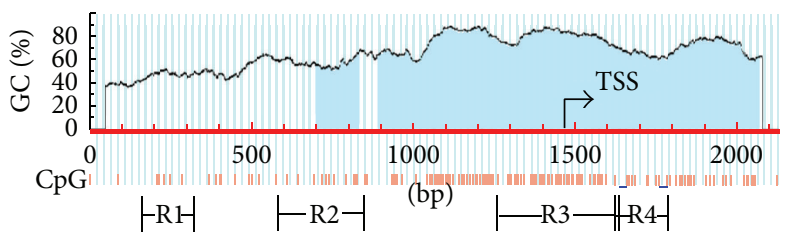

(a)
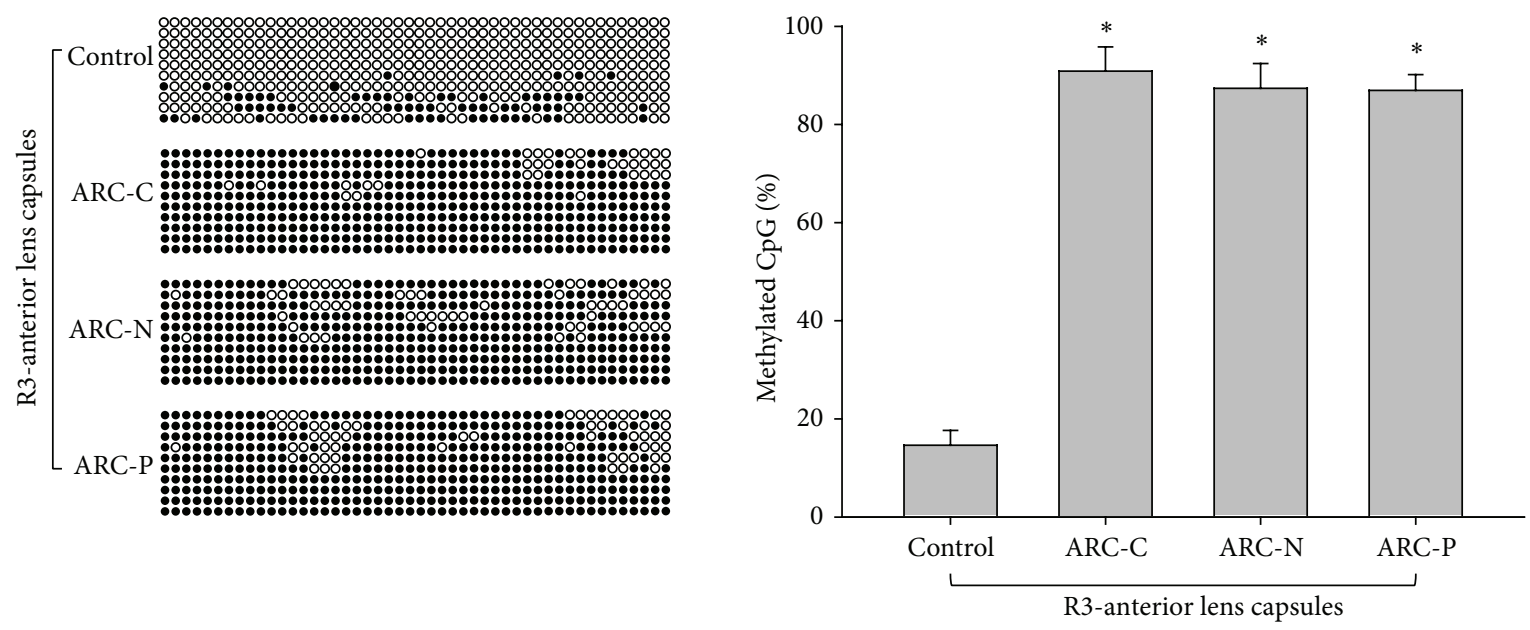

(b)
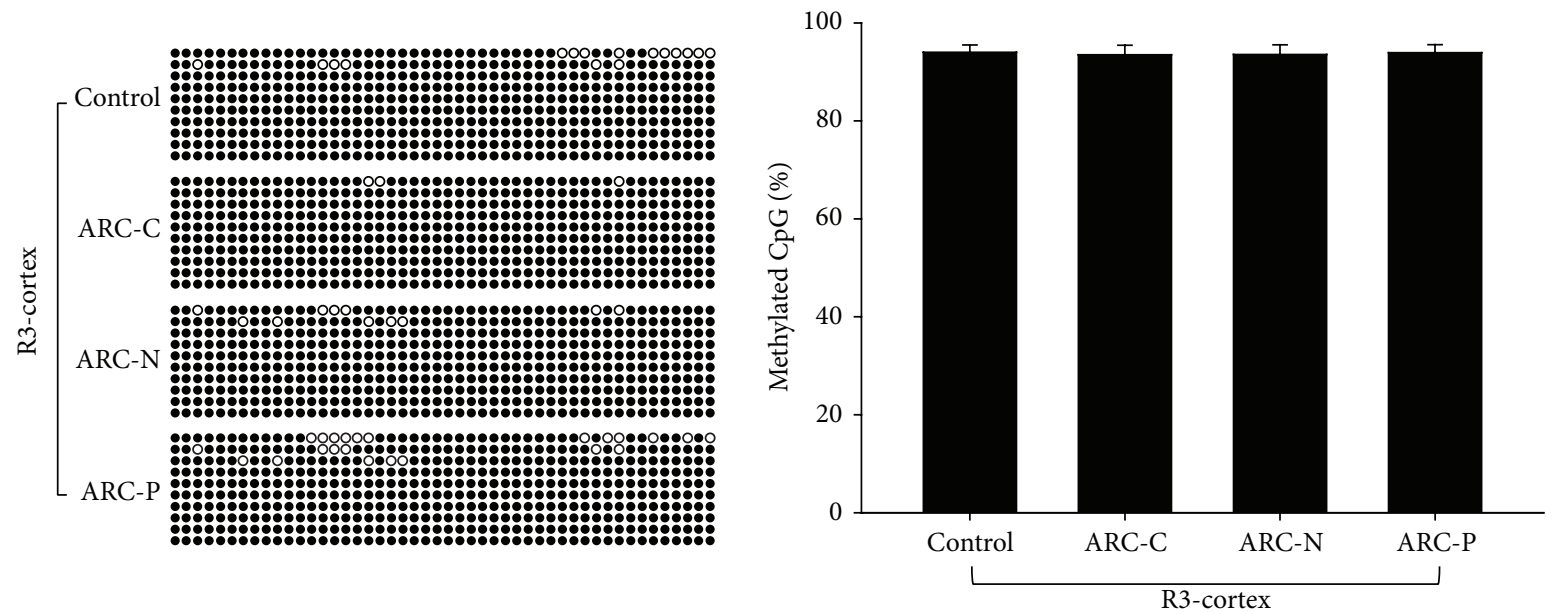

(c)

Figure 2: Methylation status at region 3 in WRN promoter in anterior lens capsules and cortex of control and ARC. (a) The positions of CpG islands within WRN promoter. In the following panels, each row of circles represents a single clone. Open and close circles represent unmethylated and methylated CpG sites, respectively. (b) Methylation status of region 3 in WRN promoter in anterior lens capsules of control $(n=20)$ and ARC-C $(n=20)$, ARC-N $(n=20)$, and ARC-P $(n=20)$. The ARC group displayed hypermethylation compared to the control group. ${ }^{*} P<0.01$. (c) Methylation status of region 3 in WRN promoter in lens cortex of control and ARC lens. There were no significant statistical differences between the ARC group and the control group. $P>0.05$.

on the DNA repair mechanism in the pathogenesis of ARC, and the knowledge can be used to identify novel options for the prevention and therapy for ARC.

\section{Disclaimer}

The authors are responsible for the content and writing of the paper.

\section{Conflict of Interests}

The authors declare no conflict of interests.

\section{Acknowledgments}

This study was supported by the National Natural Science Foundation of China (no. 81270987 and no. 81070718), the 333 


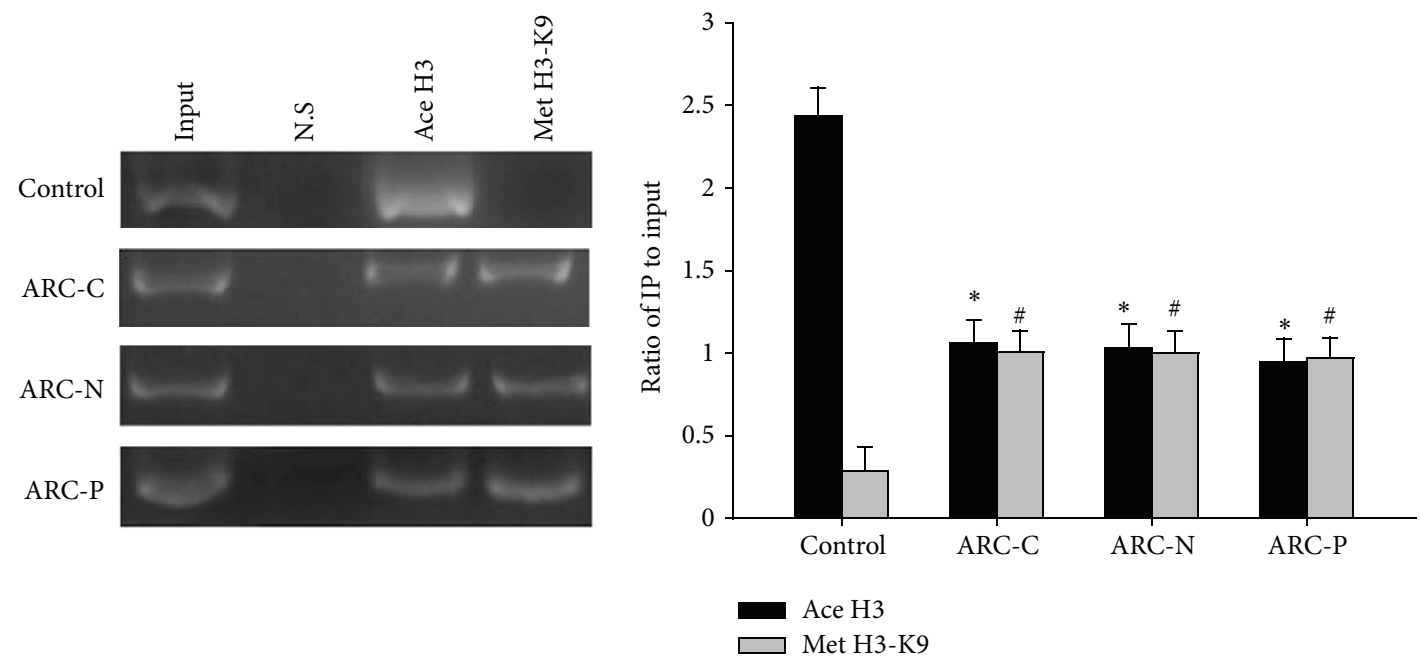

FIGURE 3: Histone modifications of the WRN promoter in anterior lens capsules of control and ARC. In anterior lens capsules, compared with the control group $(n=10)$, ChIP analysis revealed that acetylated H3 levels were lower in ARC-C $(n=10), \mathrm{ARC}-\mathrm{N}(n=10)$, and ARC-P $(n=10)$. At the same time, methylated H3-K9 was increased. ${ }^{*} P<0.01$.
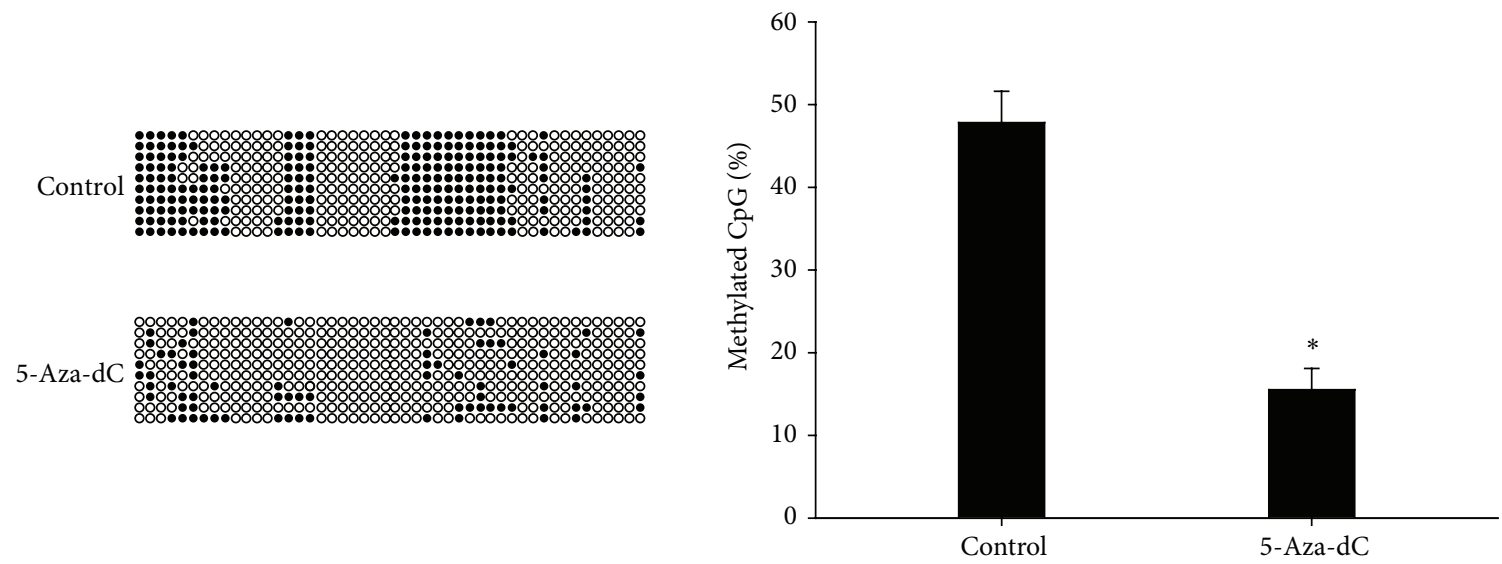

(a)
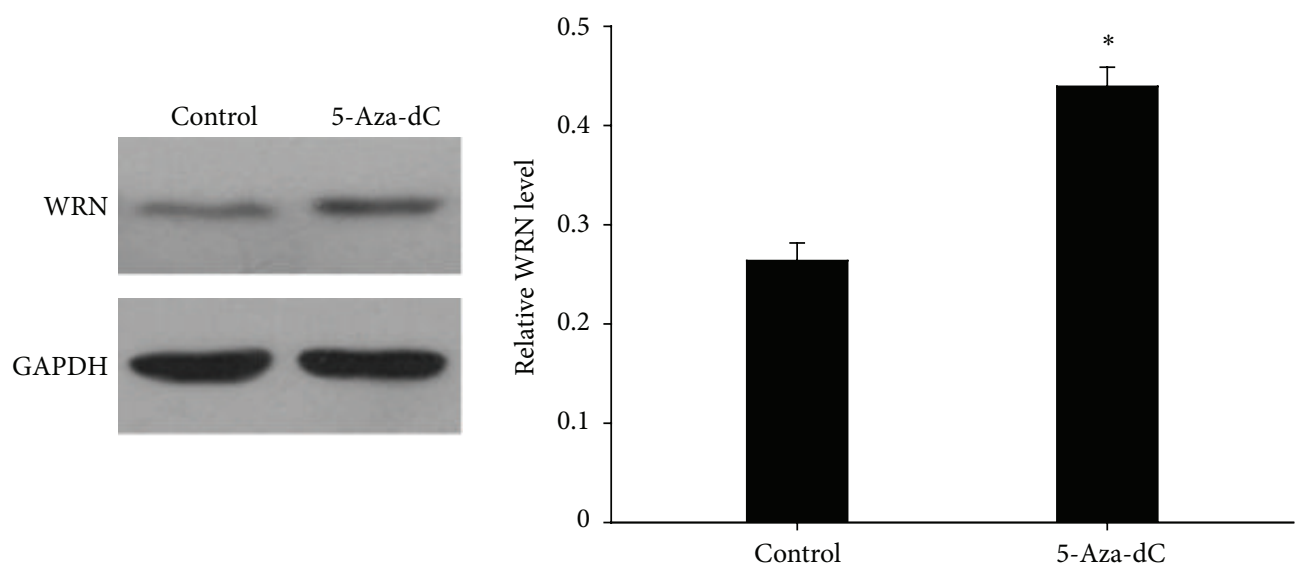

(b)

FIGURE 4: Relative methylation status and expression of protein levels of WRN in HLEB-3 after treatment with 5-aza-dC. In the following panels, each row of circles represents a single clone. Open and close circles represent unmethylated and methylated CpG sites, respectively. (a) After treatment with 5-aza-dC, the methylation rate of WRN in HLEB-3 was decreased. (b) Protein expression of WRN in the untreated control cells and in cells after treatment with 5 -aza-dC. Relative WRN protein level to GAPDH is presented as mean \pm SD. ${ }^{*} P<0.01$. 
Project of Jiangsu Province, China (no. BRA2010173), and the Ordinary University Graduate Student Research Innovation project of Jiangsu Province, China (no. 03050487).

\section{References}

[1] B. Thylefors, "A global initiative for the elimination of avoidable blindness," The American Journal of Ophthalmology, vol. 125, no. 1, pp. 90-93, 1998.

[2] B. E. K. Klein, R. Klein, and K. L. P. Linton, "Prevalence of age-related lens opacities in a population: the Beaver Dam Eye Study," Ophthalmology, vol. 99, no. 4, pp. 546-552, 1992.

[3] J. Zuercher, J. Neidhardt, I. Magyar et al., "Alterations of the 5/untranslated region of SLC16A12 lead to age-related cataract," Investigative Ophthalmology \& Visual Science, vol. 51, no. 7, pp. 3354-3361, 2010.

[4] A. Shiels and J. F. Hejtmancik, "Genetic origins of cataract," Archives of Ophthalmology, vol. 125, no. 2, pp. 165-173, 2007.

[5] C. J. Hammond, H. Snieder, T. D. Spector, and C. E. Gilbert, "Genetic and environmental factors in age-related nuclear cataracts in monozygotic and dizygotic twins," The New England Journal of Medicine, vol. 342, no. 24, pp. 1786-1790, 2000.

[6] S. Ottonello, C. Foroni, A. Carta, S. Petrucco, and G. Maraini, "Oxidative stress and age-related cataract," Ophthalmologica, vol. 214 , no. 1, pp. 78-85, 2000.

[7] O. Ates, H. H. Alp, I. Kocer, O. Baykal, and I. A. Salman, "Oxidative DNA damage in patients with cataract," Acta Ophthalmologica, vol. 88, no. 8, pp. 891-895, 2010.

[8] R. J. W. Truscott, "Age-related nuclear cataract-oxidation is the key," Experimental Eye Research, vol. 80, no. 5, pp. 709-725, 2005.

[9] W. Pendergrass, P. Penn, D. Possin, and N. Wolf, "Accumulation of DNA, nuclear and mitochondrial debris, and ROS at sites of age-related cortical cataract in mice," Investigative Ophthalmology and Visual Science, vol. 46, no. 12, pp. 4661-4670, 2005.

[10] Y. Zhang, L. Zhang, L. Zhang, J. Bai, H. Y. Ge, and P. Liu, "Expression changes in DNA repair enzymes and mitochondrial DNA damage in aging rat lens," Molecular Vision, vol. 16, pp. 1754-1763, 2010.

[11] L. Kang, W. Zhao, G. Zhang, and H. Guan, "Acetylated 8oxoguanine DNA glycosylase 1 and its relationship with p300 and SIRT1 in lens epithelium cells from age-related cataract," Experimental Eye Research, 2015.

[12] B. Xu, L. Kang, G. Zhang et al., "The changes of 8-OHdG, hOGG1, APE1 and Pol beta in lenses of patients with age-related cataract," Current Eye Research, vol. 40, no. 4, pp. 378-385, 2015.

[13] R. D. Wood, M. Mitchell, J. Sgouros, and T. Lindahl, "Human DNA repair genes," Science, vol. 291, no. 5507, pp. 1284-1289, 2001.

[14] D. M. Wilson III, T. M. Sofinowski, and D. R. McNeill, "Repair mechanisms for oxidative DNA damage," Frontiers in Bioscience, vol. 8, pp. D963-D981, 2003.

[15] D. K. Singh, B. Ahn, and V. A. Bohr, "Roles of RECQ helicases in recombination based DNA repair, genomic stability and aging," Biogerontology, vol. 10, no. 3, pp. 235-252, 2009.

[16] H. Yan, J. McCane, T. Toczylowski, and C. Y. Chen, "Analysis of the Xenopus Werner syndrome protein in DNA double-strand break repair," The Journal of Cell Biology, vol. 171, no. 2, pp. 217227, 2005

[17] J. Gee, Q. X. Ding, and J. N. Keller, "Analysis of Werner's expression within the brain and primary neuronal culture," Brain Research, vol. 940, no. 1-2, pp. 44-48, 2002.
[18] L. H. Camacho, A. Mora-Bowen, R. Munden, W. R. Smythe, and N. G. Ordoñez, "Malignant mesothelioma: natural history, pathologic features and future therapies," American Journal of Medicine, vol. 120, no. 7, pp. E7-E9, 2007.

[19] M. Goto, "Hierarchical deterioration of body systems in Werner's syndrome: implications for normal ageing," Mechanisms of Ageing and Development, vol. 98, no. 3, pp. 239-254, 1997.

[20] S. Su, Y. Yao, R. R. Zhu et al., “The associations between single nucleotide polymorphisms of DNA repair genes, DNA damage, and age-related cataract: Jiangsu eye study," Investigative Ophthalmology and Visual Science, vol. 54, no. 2, pp. 1201-1207, 2013.

[21] J. Jiang, J. Zhou, Y. Yao et al., "Copy number variations of DNA repair genes and the age-related cataract: jiangsu eye study," Investigative Ophthalmology \& Visual Science, vol. 54, no. 2, pp. 932-938, 2013.

[22] M. M. Liu, C. C. Chan, and J. S. Tuo, "Epigenetics in ocular diseases," Current Genomics, vol. 14, no. 3, pp. 166-172, 2013.

[23] T. Jenuwein and C. D. Allis, "Translating the histone code," Science, vol. 293, no. 5532, pp. 1074-1080, 2001.

[24] J. C. Rice and B. W. Futscher, "Transcriptional repression of BRCA1 by aberrant cytosine methylation, histone hypoacetylation and chromatin condensation of the BRCA1 promoter," Nucleic Acids Research, vol. 28, no. 17, pp. 3233-3239, 2000.

[25] J. A. Fahrner, S. Eguchi, J. G. Herman, and S. B. Baylin, "Dependence of histone modifications and gene expression on DNA hypermethylation in cancer," Cancer Research, vol. 62, no. 24, pp. 7213-7218, 2002.

[26] S. Fujii, R. Z. Luo, J. Yuan et al., "Reactivation of the silenced and imprinted alleles of ARHI is associated with increased histone $\mathrm{H} 3$ acetylation and decreased histone H3 lysine 9 methylation," Human Molecular Genetics, vol. 12, no. 15, pp. 1791-1800, 2003.

[27] I. O. Nasonkin, K. Lazo, D. Hambright, M. Brooks, R. Fariss, and A. Swaroop, "Distinct nuclear localization patterns of DNA methyltransferases in developing and mature mammalian retina," The Journal of Comparative Neurology, vol. 519, no. 10, pp. 1914-1930, 2011.

[28] Y. Wang, F. Li, G. Zhang, L. Kang, B. Qin, and H. Guan, "Altered DNA methylation and expression profiles of 8-oxoguanine DNA glycosylase 1 in lens tissue from age-related cataract patients," Current Eye Research, 2014.

[29] M. Gemenetzi and A. J. Lotery, "The role of epigenetics in agerelated macular degeneration," Eye, vol. 28, no. 12, pp. 1407-1417, 2014.

[30] M. Beta, S. Chitipothu, V. Khetan, J. Biswas, and S. Krishnakumar, "Hypermethylation of adenomatosis polyposis coli-2 and its tumor suppressor role in retinoblastoma," Current Eye Research, pp. 1-10, 2014.

[31] R. A. Kowluru, J. M. Santos, and M. Mishra, "Epigenetic modifications and diabetic retinopathy," BioMed Research International, vol. 2013, Article ID 635284, 9 pages, 2013.

[32] L. Wei, B. Liu, J. Tuo et al., "Hypomethylation of the IL17RC promoter associates with age-related macular degeneration," Cell Reports, vol. 2, no. 5, pp. 1151-1158, 2012.

[33] R. Agrelo, W. H. Cheng, F. Setien et al., "Epigenetic inactivation of the premature aging Werner syndrome gene in human cancer," Proceedings of the National Academy of Sciences of the United States of America, vol. 103, no. 23, pp. 8822-8827, 2006.

[34] K. Futami, M. Takagi, A. Shimamoto, M. Sugimoto, and Y. Furuichi, "Increased chemotherapeutic activity of camptothecin in cancer cells by siRNA-induced silencing of WRN 
helicase," Biological and Pharmaceutical Bulletin, vol. 30, no. 10, pp. 1958-1961, 2007.

[35] K. Masuda, K. Banno, M. Yanokura et al., "Association of epigenetic inactivation of the WRN gene with anticancer drug sensitivity in cervical cancer cells," Oncology Reports, vol. 28, no. 4, pp. 1146-1152, 2012.

[36] L. Wang, L. Xie, J. Wang, J. Shen, and B. Liu, "Correlation between the methylation of SULF2 and WRN promoter and the irinotecan chemosensitivity in gastric cancer," BMC Gastroenterology, vol. 13, no. 1, article 173, 2013.

[37] G. Supic, R. Kozomara, M. Brankovic-Magic, N. Jovic, and Z. Magic, "Gene hypermethylation in tumor tissue of advanced oral squamous cell carcinoma patients," Oral Oncology, vol. 45, no. 12, pp. 1051-1057, 2009.

[38] L. T. Chylack Jr., J. K. Wolfe, D. M. Singer et al., "The lens opacities classification system III," Archives of Ophthalmology, vol. 111, no. 6, pp. 831-836, 1993. 


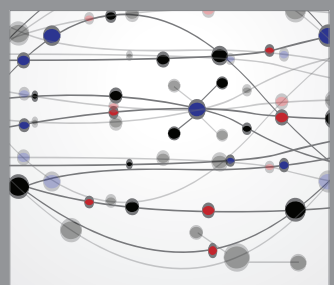

The Scientific World Journal
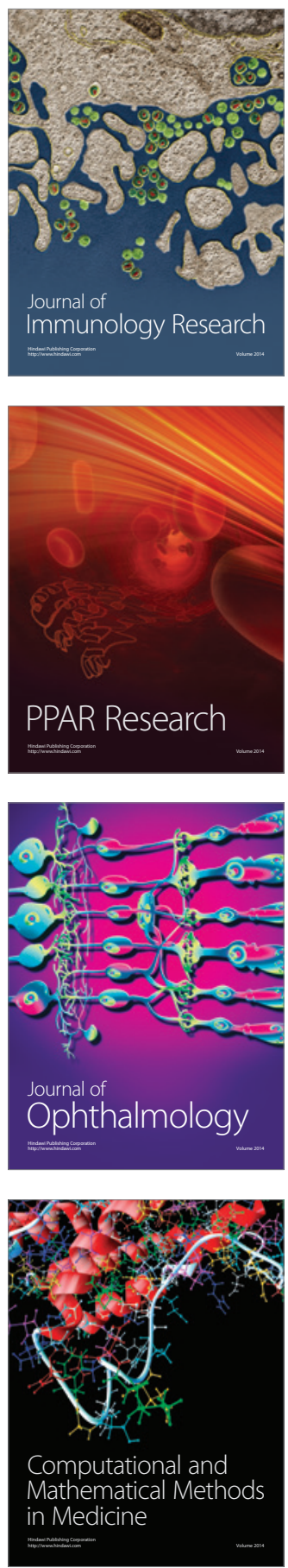

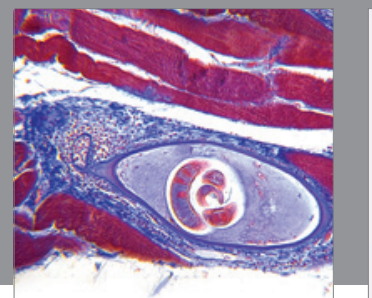

Gastroenterology

Research and Practice
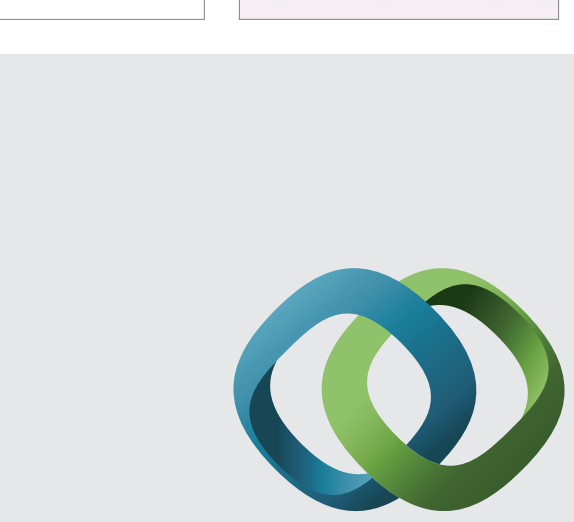

\section{Hindawi}

Submit your manuscripts at

http://www.hindawi.com
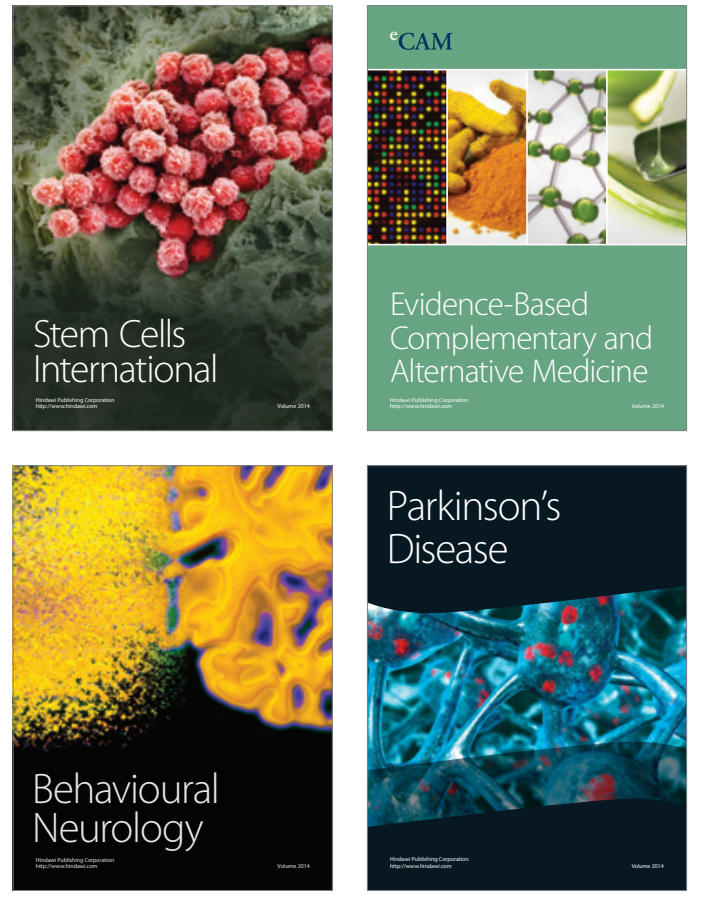
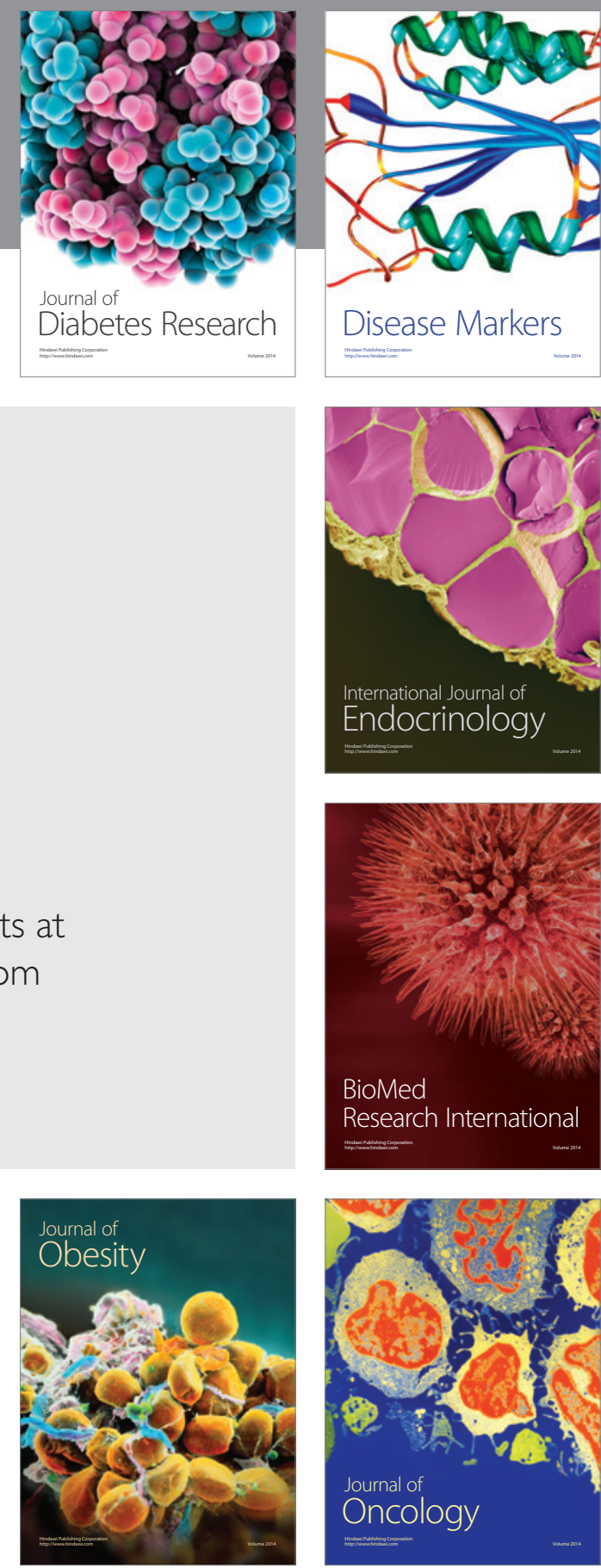

Disease Markers
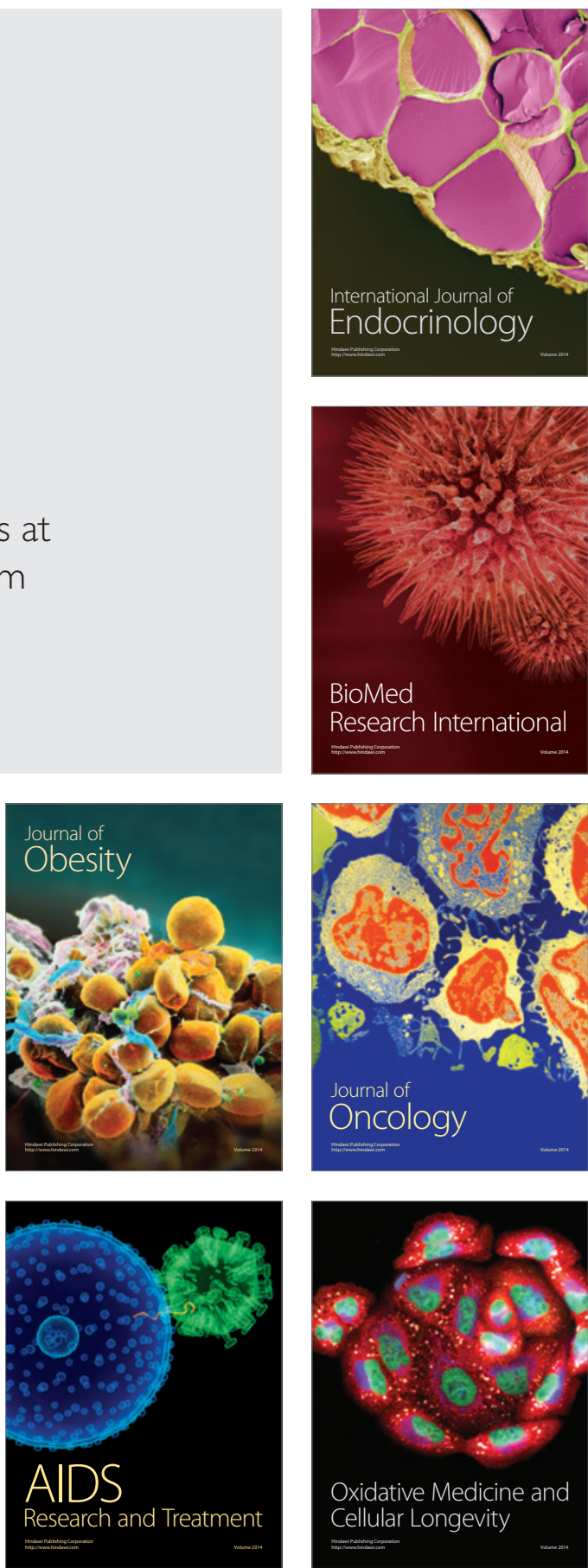